\title{
GIC Based Third-order Active Low-pass Filters
}

\author{
Nino Stojković, Dražen Jurišić and Neven Mijat \\ University of Zagreb, Faculty of Electrical Engineering and Computing \\ Unska 3, 10000 Zagreb, Croatia \\ Tel. +38516129 911,Fax. +38516129652,e-mail:nino.stojkovic@fer.hr
}

\begin{abstract}
Third order low-pass filters are analyzed. Two different configuration of General Impedance Converter (GIC) based filters are compared to two Sallen and Key (SAK) based structures. For GIC based third-order filters realization procedures are given. The transfer functions and the component values for the third-order filters with Butterworth response are presented. The sensitivity analysis is done, and the results of Monte Carlo runs, as well as Schoeffler sensitivities are shown. The best results are obtained with the GIC based filter, which use four capacitances for the realization.
\end{abstract}

\section{Introduction}

Many practical filtering problems in electronic design can be solved by low cost, low order, easy to realize active filters. Second- and/or third-order active filter sections are usually suitable enough for such applications. Various second-order single or multiple amplifier filter sections are widely used as building blocks of high-order filters as well, and significant efforts in the past have been paid to their analysis and design [1]. On the other hand third-order filter sections attracted considerably less attention of filter designers, mostly thanking to the fact that their application in high-order filters is rather unusual. It is well known that high-order LP or HP filters are usually realized as a cascade of second-order blocks, if the order $n$ of a filter is even, and one first-order section for odd filter transfer function order. The properties of an odd-order filter can be improved if one second-order section and a first-order section are replaced by some less sensitive third-order section. Some authors have even proposed the approximation technique suitable for realization of highorder filters by cascading third-order filter blocks [2].

Third-order filter structures commonly used in filter realizations, are considered as single-amplifier configurations, which are suitable from many points such as the power consumption, ease of realization procedure, low cost etc. In this paper we introduce a less sensitive two-amplifier third-order active filter sections, which can be applied for low-order filtering applications, as well as for above mentioned replacement in high-order filters. The filter sections are based on a general impedance converter (GIC) circuit used for realizations of second-order filter blocks [3].

\section{Third-order low-pass filters}

GIC based second-order active filter sections are well known low sensitive filter structures, suitable for realization of high-order filters. The third-order low-pass (LP) filters can be easily realized by cascading such sections with the first-order LP filter realized by a simple $\mathrm{RC}$ circuit and a voltage follower. By connecting the RC circuit without the voltage follower, to the input of a second-order GIC based filter section, a third-order section can be realized using one of the filter structures shown in Fig. 1.a) and 1.b) denoted as GIC1 and GIC2 respectively. For such circuit new values of filter components must be calculated. The corresponding realization procedures are presented in the sequel, and compared to the Sallen and Key based third-order filters shown in Fig 1.c) and 1.d) denoted by SAK1 and SAK2, [1], [4].

\subsection{Third-order GIC based filter with 3 capacitances (GIC1)}

The first GIC based third-order filter which is analyzed is a filter structure with three capacitances (GIC1), shown in Fig. 1.a). The transfer function is shown in Table 1. Calculation of the filter elements can be performed by comparison of the transfer function of GIC1 to the general LP third-order transfer function

$$
T(s)=\frac{b_{0}}{s^{3}+a_{2} s^{2}+a_{1} s+a_{0}},
$$

giving the following identities

$$
\frac{G_{0}+G_{6}}{C_{0}}=a_{2}
$$



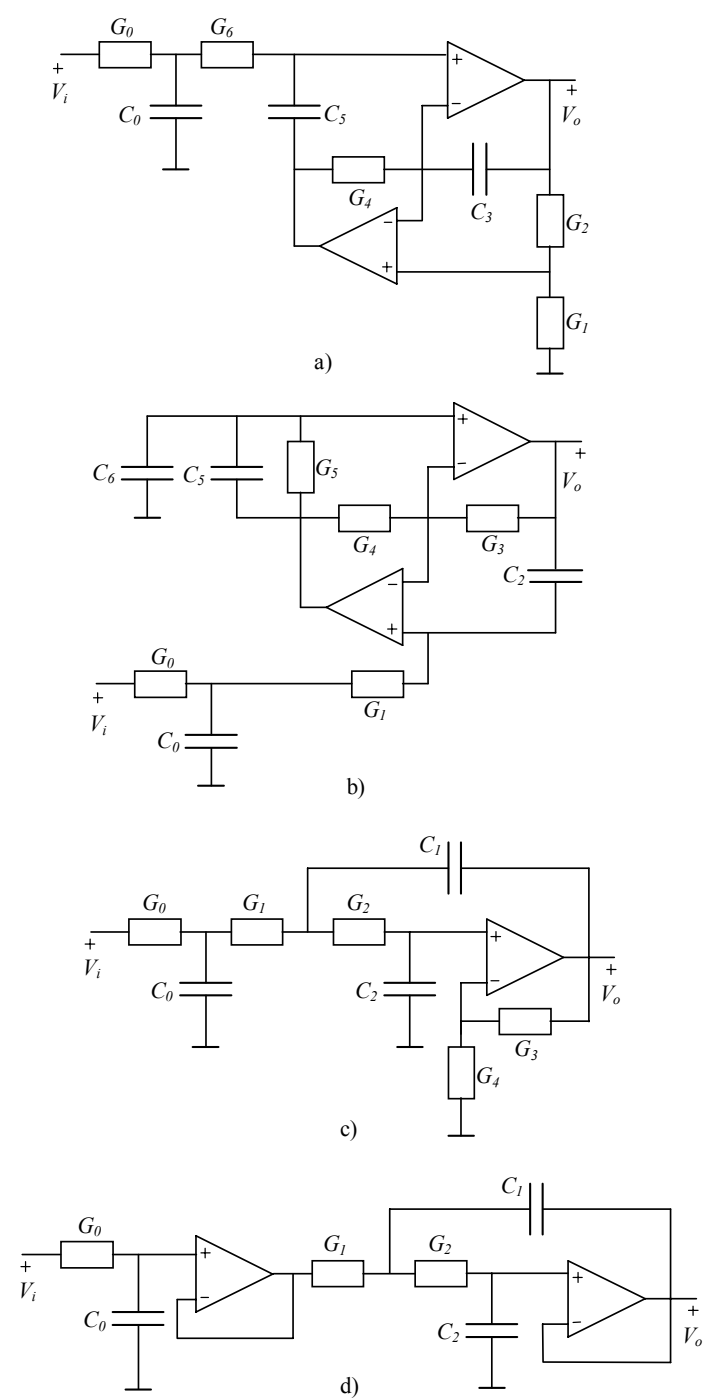

Figure 1. Third-order active filters: a) GIC1, b) GIC2, c) SAK1, d) SAK2

$$
\begin{aligned}
& \frac{G_{2} G_{4} G_{6}}{G_{1} C_{3} C_{5}}=a_{1} \\
& \frac{G_{0} G_{2} G_{4} G_{6}}{G_{1} C_{0} C_{3} C_{5}}=a_{0} \\
& \left(1+\frac{G_{2}}{G_{1}}\right) \frac{G_{0} G_{4} G_{6}}{C_{0} C_{3} C_{5}}=b_{0} .
\end{aligned}
$$

Since there are 4 equations with 8 unknowns, the additional conditions are introduced

$$
C_{3}=C_{5}=C, \quad G_{4}=G_{6}=G,
$$

and the components can be expressed as functions of capacitance $\mathrm{C}$.

$$
\begin{aligned}
& G=C \sqrt{a_{1}} \\
& G_{0}=C \frac{b_{0} \sqrt{a_{1}}}{a_{2} a_{1}-b_{0}}
\end{aligned}
$$

$$
\begin{aligned}
& C_{0}=C \frac{\sqrt{a_{1}}}{a_{2}-\frac{b_{0}}{a_{1}}} \\
& G_{1}=G_{2}
\end{aligned}
$$

Choosing initial value for the capacitance $\mathrm{C}$ all elements are defined except the resistors $G_{I}$ and $G_{2}$, which can be chosen freely. The calculated element values for the GIC1 third-order Butterworth low-pass filter are presented in Table 2 .

\subsection{Third-order GIC based filter with 4 capacitances (GIC2)}

Another configuration of the GIC based third-order low-pass filter (GIC2), needs four capacitances for the realization as shown in Fig. 1.b). The transfer function of this filter is shown in Table 1. It can be obtained only if the condition

$$
C_{5} G_{3}=C_{6} G_{4} \quad,
$$

is satisfied. After comparison of the GIC2 transfer function to the general low-pass third-order transfer function (1) the following equations are obtained:

$$
\begin{aligned}
& \frac{G_{0}+G_{1}}{C_{0}}+\frac{G_{1}}{C_{2}}=a_{2} \\
& \frac{G_{0} G_{1}}{C_{0} C_{2}}+\frac{G_{1} G_{5}}{C_{2} C_{5}}=a_{1} \\
& \frac{G_{0} G_{1} G_{5}}{C_{0} C_{2} C_{5}}=a_{0} \\
& \frac{G_{0} G_{1} G_{5}}{C_{0} C_{2} C_{5}}=b_{0}
\end{aligned}
$$

In order to simplify the calculation procedure we introduce a new variable $\tau_{0}$ :

$$
\tau_{0}=\frac{C_{0}}{G_{0}}
$$

It can be shown that $\tau_{0}$ must be in range

$$
-\frac{1}{\sigma}<\tau_{0}<\frac{a_{1}}{a_{0}}
$$

where $\sigma$ represents negative real pole of the third-order transfer function (1) and $\mathrm{a}_{1}$ and $\mathrm{a}_{2}$ are the denominator coefficients of the same function. Choosing capacitances $\mathrm{C}_{0}$ and $\mathrm{C}_{5}$ the rest of filter elements can be calculated as follows:

$$
\begin{aligned}
& G_{0}=\frac{C_{0}}{\tau_{0}} \\
& G_{1}=G_{0}\left(a_{2} \tau_{0}-a_{1} \tau_{0}^{2}+a_{0} \tau_{0}^{3}-1\right) \\
& C_{2}=G_{1} \frac{1}{a_{1} \tau_{0}-a_{0} \tau_{0}^{2}}
\end{aligned}
$$




$$
G_{5}=C_{5} \frac{a_{0}}{a_{1}-a_{0} \tau_{0}}
$$

Setting

$$
G_{3}=G_{4}
$$

from (11) follows

$$
C_{6}=C_{5}
$$

Calculated element values for the GIC2 third-order Butterworth low-pass filter are presented in Table 2.

\subsection{Third-order SAK based filters (SAK1 and SAK2)}

The GIC based filters are compared to SAK based filters. For that purpose, the third-order low-pass SAK filter with one operational amplifier, shown in Fig. 1.c) and with two operational amplifiers shown in Fig. 1.d) are analyzed. Transfer functions of these filters are shown in Table 1. and calculated elements for the filter with Butterworth response are given in Table 2.

\section{Sensitivity analysis}

In order to compare the influence of filter parameters variations to the filter amplitude response for various structures, the sensitivities of the filter amplitude response $|T(j \omega)|$ to the variation of elements $x_{i}$ are analyzed. The sensitivity function $\mathrm{S}$ is defined with

$$
S_{x_{i}}^{|T(j \omega)|}=\frac{d|T(j \omega)|}{d x_{i}} \cdot \frac{x_{i}}{|T(j \omega)|}
$$

If the gain of the amplitude response $\alpha(\omega)$ is expressed by

$$
\alpha(\omega)=20 \log |T(j \omega)|[\mathbf{d B}],
$$

the gain sensitivity function $S$ can be defined as

$$
\mathbf{s}_{x_{i}}^{\alpha(\omega)}=x_{i} \frac{d \alpha(\omega)}{d x_{i}}[d B]
$$

We can define the gain variation as

$$
\Delta \alpha(\omega) \cong \sum_{i} \mathbf{S}_{x_{i}}^{\alpha(\omega)} \frac{\Delta x_{i}}{x_{i}}[d B] \quad,
$$

where $x_{i}$ represents all passive filter elements. As a sensitivity measure the Schoeffler multiparameter sensitivity function $\mathrm{I}_{\mathrm{s}}^{2}(\omega)$

$$
I_{s}^{2}(\omega)=\sum_{i}\left(\mathbf{s}_{x_{i}}^{\alpha(\omega)}\right)^{2}
$$

is used [5]. It can be shown that if all network component variations are uncorrelated random variables with zero expectations and the same standard deviation $\sigma$, than the standard deviation of gain variation is approximately

\begin{tabular}{|c|c|}
\hline & $T(s)=\frac{V_{o}(s)}{V_{i}(s)}$ \\
\hline \multirow[t]{2}{*}{ GIC1 } & $\left(1+\frac{G_{2}}{G_{1}}\right) \frac{G_{0} G_{4} G_{6}}{C_{0} C_{3} C_{5}}$ \\
\hline & $s^{3}+s^{2}\left(\frac{G_{0}+G_{6}}{C_{0}}\right)+s\left(\frac{G_{2} G_{4} G_{6}}{G_{1} C_{3} C_{5}}\right)+\frac{G_{0} G_{2} G_{4} G_{6}}{G_{1} C_{0} C_{3} C_{5}}$ \\
\hline \multirow{3}{*}{ GIC2 } & $G_{0} G_{1} G_{5}$ \\
\hline & $\frac{C_{0} C_{2} C_{5}}{,}, C_{5} G_{3}=C_{6} G_{4}$ \\
\hline & $s^{3}+s^{2}\left(\frac{G_{0}+G_{1}}{C_{0}}+\frac{G_{1}}{C_{2}}\right)+s\left(\frac{G_{0} G_{1}}{C_{0} C_{2}}+\frac{G_{1} G_{5}}{C_{2} C_{5}}\right)+\frac{G_{0} G_{1} G_{5}}{C_{0} C_{2} C_{5}}$ \\
\hline \multirow[t]{2}{*}{ SAK1 } & $\left(1+\frac{G_{4}}{G_{3}}\right) \frac{G_{0} G_{1} G_{2}}{C_{0} C_{1} C_{2}}$ \\
\hline & $s^{3}+s^{2}\left(\frac{G_{0}+G_{1}}{C_{0}}+\frac{G_{1}+G_{2}}{C_{1}}-\frac{G_{2} G_{4}}{C_{2} G_{3}}\right)+s\left(\frac{G_{0} G_{1}+G_{0} G_{2}+G_{1} G_{2}}{C_{0} C_{1}}+\frac{G_{1} G_{2}}{C_{1} C_{2}}-\frac{G_{0} G_{2} G_{4}+G_{1} G_{2} G_{4}}{C_{0} C_{2} G_{3}}\right)+\frac{G_{0} G_{1} G_{2}}{C_{0} C_{1} C_{2}}$ \\
\hline \multirow{2}{*}{ SAK2 } & $\frac{G_{0} G_{1} G_{2}}{C_{0} C_{1} C_{2}}$ \\
\hline & $s^{3}+s^{2}\left(\frac{G_{0}}{C_{0}}+\frac{G_{1}+G_{2}}{C_{1}}\right)+s\left(\frac{G_{0} G_{1}+G_{0} G_{2}}{C_{0} C_{1}}+\frac{G_{1} G_{2}}{C_{1} C_{2}}\right)+\frac{G_{0} G_{1} G_{2}}{C_{0} C_{1} C_{2}}$ \\
\hline
\end{tabular}
proportional to $\mathrm{I}_{\mathrm{s}}(\omega)$. Therefore from

$$
\sigma\left(\Delta x_{i} / x_{i}\right)=\delta
$$

it follows

$$
\sigma(\Delta \alpha(\omega)) \cong \delta I_{S}(\omega)
$$

Table 1. Transfer functions of presented third-order filters 
Table 2. Filter elements of analyzed 3rd-order filters

\begin{tabular}{|c|c|c|c|c|}
\hline & $\mathrm{GIC} 1$ & GIC2 & SAK1 & SAK2 \\
\hline $\mathrm{G}_{0}$ & 0.471405 & 0.638897 & 0.638897 & 1 \\
\hline $\mathrm{G}_{1}$ & 1.414214 & 0.680551 & 0.680552 & 1 \\
\hline $\mathrm{G}_{2}$ & 1.414214 & & 2.299900 & 1 \\
\hline $\mathrm{G}_{3}$ & & 1 & 1 & \\
\hline $\mathrm{G}_{4}$ & 1.414214 & 1 & 1 & \\
\hline $\mathrm{G}_{5}$ & & 2.299899 & & \\
\hline $\mathrm{G}_{6}$ & 1.414214 & & & \\
\hline $\mathrm{C}_{0}$ & 0.942809 & 1 & 1 & 1 \\
\hline $\mathrm{C}_{1}$ & & & 1 & 1 \\
\hline $\mathrm{C}_{2}$ & & 1 & 1 & 1 \\
\hline $\mathrm{C}_{3}$ & 1 & & & \\
\hline $\mathrm{C}_{5}$ & 1 & 1 & & \\
\hline $\mathrm{C}_{6}$ & & 1 & & \\
\hline
\end{tabular}

The standard deviation of amplitude response according to (30) is calculated assuming $1 \%$ standard deviations of elements. The results are obtained using programming tool Mathematica and shown in Fig. 2. Schoeffler sensitivity function gives results shown in Fig. 3.

The sensitivities can be compared numerically, as well, if multiparameter measure $\mathrm{M}$ defined by

$$
M=\int_{0.01}^{10} \sum_{i}\left(S_{x_{i}}^{|T(j \omega)|}\right)^{2} d \omega
$$

is used [4]. It represents the area below the correspondent sensitivity function in Fig. 3. The results are given in the Fig. 3. for all filters which have been considered. The result shows significant improvement in sensitivities for GIC based filters. Significant part of the result is placed left from the cut-off frequency, in the pass-band. In the stop-band, the sensitivities are less important because of the filter attenuation. The significant influence on signals can be done in the pass-band where amplitude response variation should be the smallest. It can be seen that the number $\mathrm{M}$ gives picture of merit, which is not completely reliable. Although the smallest $\mathrm{M}$ is obtained for GIC1 filter, in the pass-band the lowest sensitivities has GIC2 filter. The worst sensitivities has SAK1 filter. Configuration GIC1 shows good results in the neighborhood of the cut-off frequency but in pass-band its sensitivities are close to the sensitivities of SAK1 and SAK2 filters.

The same relations between the sensitivities of the considered third-order filters are obtained using Monte Carlo analysis, Fig. 4. This analysis completes filter sensitivity picture. Assuming that the component values have some finite tolerances, the amplitude response is calculated. In the presented analysis the components are assumed to have $1 \%$ tolerance. The obtained results confirm previously described relations between presented filters.

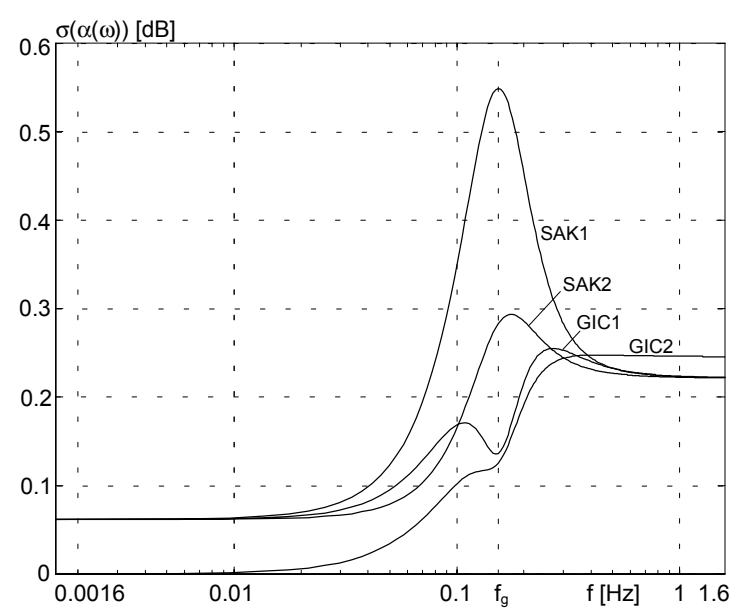

Figure 2. Standard deviation of the gain $\sigma(\alpha(\omega))$ for the filters presented in Fig. 1.

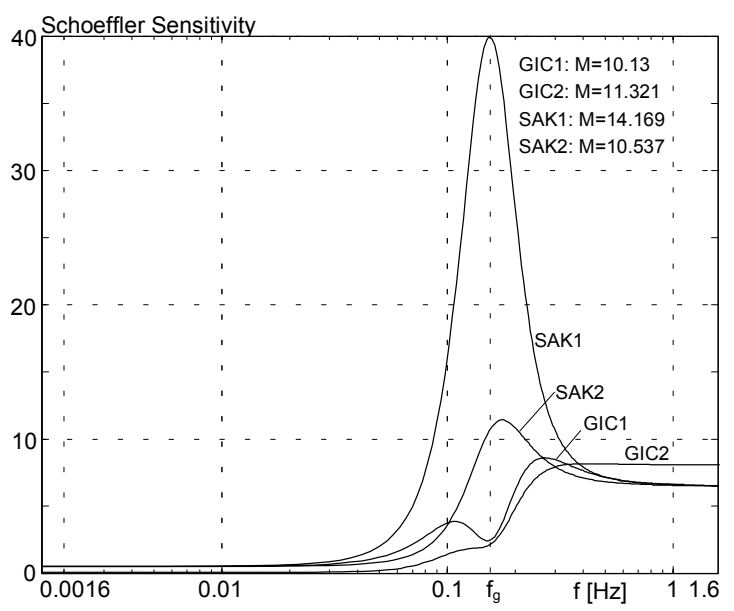

Figure 3. Schoeffler sensitivity for the filters presented in Fig. 1.

\section{Conclusions}

In this paper, third-order LP filters, which can be used for low-order, low-cost applications, as well as building blocks for high-order applications, are presented and analyzed. It is shown that low sensitivities of the filter amplitude response can be obtained by using GIC based third-order structures instead of single-amplifier SAK based configuration.

Disadvantage of GIC based filters can be the use of two operational amplifiers resulting with increased power consumption in comparison to single amplifier structures. Therefore these filter configurations should be used in the applications where power consumption is not the primary interest. The design procedure is straight-forward, and relatively simple. The sensitivity analysis is performed using Schoeffler sensitivity measure and confirmed by Monte-Carlo runs. 
Figure 4. Monte Carlo response plots of the filters presented in Fig. 1.
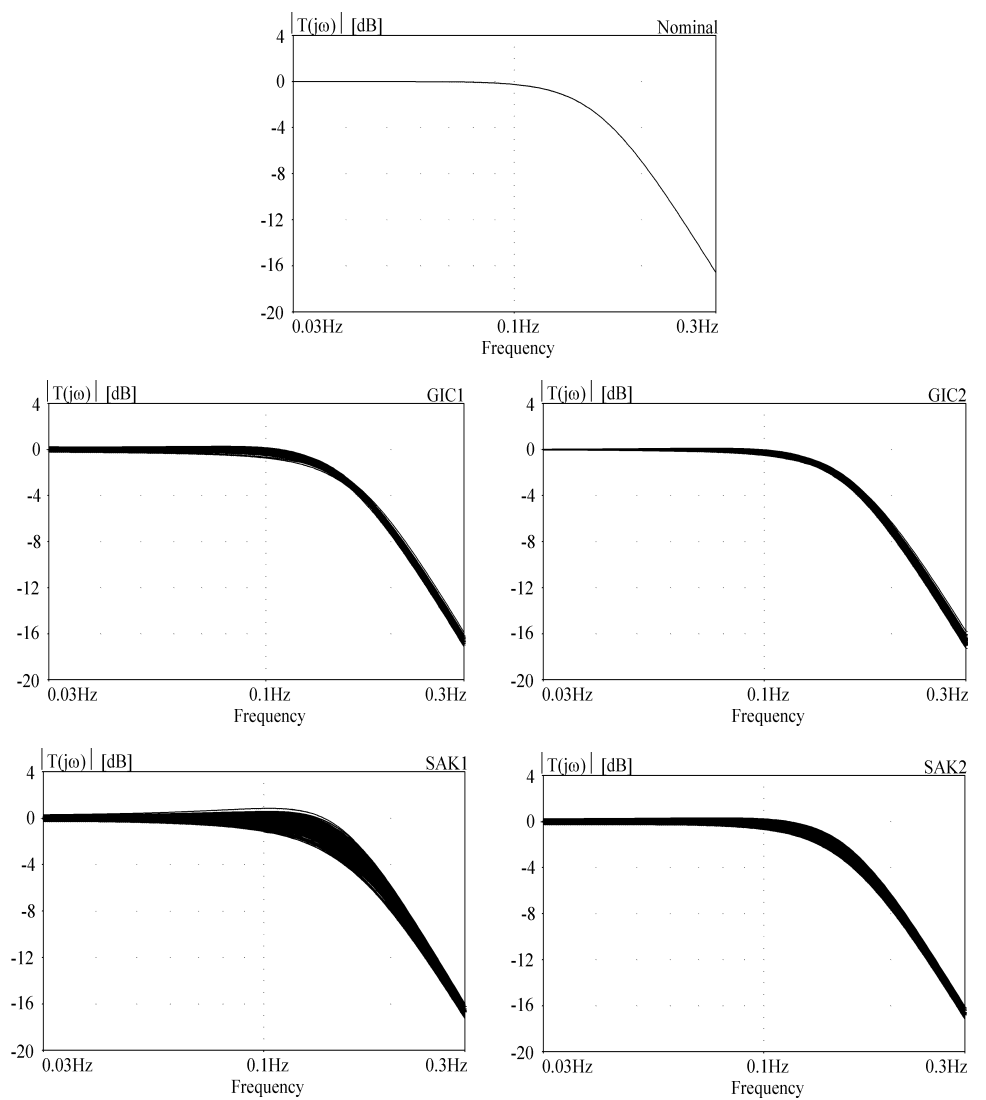

\section{References}

[1] G.S. Moschytz, Linear Integrated Networks: Design, New York, Van Nostrand-Reinhold, 1975.

[2] M. Biey, A. Premoli, Design of Low-Pass Maximally Flat $R C$ active filters with Multiple Real Pole:The MURROMAF Polynomials, IEEE Transactions on Circuits and Systems, vol. CAS-25, no. 4, April 1978.

[3] N. Fliege, A New Class of Second-order RC-Active Filters with Two Operational Amplifiers, Nachrichtentech Zeitung, Vol. 26, pp. 279-282, June 1973.

[4] R. Schauman, M. S. Ghausi, K. R. Laker, Design of Analog Filters - Passive, Active RC and Switched Capacitor, Prentice-Hall, Inc., Englewood Cliffs, New Jersey, 1990.

[5] J.D. Schoeffler, The Synthesis of Minimum Sensitivity Networks, IEEE Transactions on Circuit Theory, pp. 271276, June 1964. 